\title{
Editorial
}

\section{Advanced Signal Processing and Pattern Recognition Methods for Biometrics}

\author{
Nikolaos V. Boulgouris, ${ }^{1}$ Juwei Lu, ${ }^{2}$ Konstantinos N. Plataniotis, ${ }^{3}$ and Arun Ross ${ }^{4}$ \\ ${ }^{1}$ Department of Electronic Engineering, Division of Engineering, King's College London, London WC2R 2LS, UK \\ ${ }^{2}$ Vidient Systems, Inc. 4000 Burton Drive, Santa Clara, CA 95054, USA \\ ${ }^{3}$ The Edward S. Rogers Sr. Department of Electrical and Computer Engineering, University of Toronto, Toronto, \\ Ontario, Canada M5S $3 G 4$ \\ ${ }^{4}$ Lane Department of Computer Science and Electrical Engineering, West Virginia University, Morgantown, WV 26506, USA
}

Correspondence should be addressed to Nikolaos V. Boulgouris, nikolaos.boulgouris@kcl.ac.uk

Received 23 April 2008; Accepted 23 April 2008

Copyright (c) 2008 Nikolaos V. Boulgouris et al. This is an open access article distributed under the Creative Commons Attribution License, which permits unrestricted use, distribution, and reproduction in any medium, provided the original work is properly cited.

Biometric systems establish the identity of an individual based on physical characteristics, such as fingerprint, face, hand, and iris, or behavioral characteristics, such as gait, signature, handwriting, and keystroke dynamics. Interest in biometrics has grown significantly over the last three decades. Consequently, both academic as well as corporate research units have devoted plenty of resources to the study, research, and development of accurate and cost-effective biometric systems. Biometric authentication systems are now being used in a wide range of applications, including border security, automated surveillance, physical access control, and network security.

A typical biometric system consists of a sensor module for acquiring the biometric signal of an individual, a feature extraction module for extracting discriminant features from the signal, a matching module for comparing two sets of features, and a decision module for establishing the identity of the individual. In spite of the rapid advances made in the field of biometrics, there are still several challenges that need to be addressed in order to broaden the scope and deployment of biometrics. The most important ones among these include (i) accurate modeling of intrauser variations of a biometric signal; (ii) scaling the system to accommodate a large number of users (e.g., in the order of millions); (iii) protecting biometric templates and "canceling" them if needed; (iv) operating the system in an unconstrained environment involving noncooperative individuals; and (v) reinforcing the identity of an individual by consolidating the evidence of multiple biometric sources. A number of test suites have been developed, especially for face, fingerprint, iris, and voice systems, in order to gauge the matching accuracy of the state-of-the-art biometric algorithms. Results suggest that there is plenty of scope for improving the performance of biometric systems and incorporating them in diverse applications.

This special issue contains contributions covering a wide spectrum of topics in biometrics. The majority of the papers present methods for human recognition based on facial images, walking style (gait), fingerprints, signature, iris, and retina. There are also papers presenting new methods based on electrocardiogram (EEG) and electroencephalogram (EEC). Other papers discuss techniques for biometric fusion and enhancing template security. As a whole, the special issue includes a very diverse collection of high-quality contributions authored by leading researchers in the field.

The first paper, by A. K. Jain et al., presents an extensive categorization of the various vulnerabilities of a biometric system, and discusses countermeasures that have been proposed to address these vulnerabilities. Special emphasis is placed on biometric template security which is an important issue because, unlike passwords and tokens, compromised biometric templates cannot be easily revoked and reissued.

The second paper, by $Z$. Yue et al., deals with face recognition. The objective of their work is to identify a subject from a probe image acquired under different pose and illumination conditions in the presence of only a single gallery image of the subject in the database. The authors of 
this paper extend the spherical harmonics representation to encode pose information.

The next paper, by P. R. Nicholl et al., performs face recognition by combining the multiresolution feature of the discrete wavelet transform (DWT) with the local interactions of the facial structures expressed through the structural hidden Markov model (SHMM). Experimental results suggest the benefits of employing SHMMs over traditional HMMs.

The paper by Y. Xu et al. presents a framework for videobased face recognition that is robust to large changes in facial pose and lighting conditions. The proposed method advocates a novel theoretical framework that can integrate the effects of motion, lighting, and shape in generating an image using a perspective camera. The method can handle situations where the pose and lighting conditions of the training and testing data are completely different.

The paper by S.-K. Kim et al. presents a method for face recognition using ancillary information. Specifically, the authors fuse the local and global information contained within a facial image in an adaptive manner for reliable recognition. Additionally, they explicitly estimate external factors such as pose and illumination, and incorporate this information in the fusion framework. Empirical evaluations indicate that the adoption of the proposed method leads to noticeable improvements in matching performance.

The paper by J. Uglov et al. addresses the impact of noisy facial images on the performance of face recognition system. Although multiclass neural networks can be used for combating the above problems, such systems are not robust when the number of subjects is increased. The paper explores a pairwise neural network system as an alternative approach to improving the robustness of face recognition. Experiments show that the pairwise recognition system outperforms the multiclass recognition system in terms of its predictive accuracy on the test face images.

The next two papers are on gait recognition. The first paper is authored by $\mathrm{H}$. Lu et al. and presents a full-body layered deformable model (LDM) for automatic modelbased gait recognition. In this paper, algorithms for LDMbased human body pose recovery are developed to estimate the LDM parameters from both manually labeled and automatically extracted silhouettes. The estimated LDM parameters are used for model-based gait recognition. The results demonstrate that the recognition performance benefits from not only the lower-limb dynamics, but also the dynamics of the upper limbs, the shoulders, and the head. The second paper is authored by X. Huang and N. V. Boulgouris and presents a gait recognition method based on multiview gait sequences. This paper investigates the case in which several views are available for gait recognition. It is shown that each view has unequal discrimination power and, therefore, should have unequal contribution in the recognition process. A novel approach for the combination of the results from several views is also proposed based on the relative importance of each view.

I. G. Damousis et al. discuss the design of a multimodal biometric system which was carried out in the framework of a European project. The authors discuss the combination of new types of biometrics with the state-of-the-art sensor technologies in order to enhance security in a wide spectrum of applications. The objective is to develop a modular, robust, multimodal biometrics security authentication and monitoring system which utilizes a biodynamic physiological profile, unique for each individual. An additional objective is to make advancements in the state of the art in behavioral and other biometrics.

The paper by N. Balasubramanian et al. presents a method for person-independent head pose estimation. The estimation of head-pose angle is necessary and useful for several face analysis applications. In this paper, a framework is proposed for a supervised form of manifold learning called biased manifold embedding. The proposed framework goes beyond pose estimation, and can be applied to all regression applications. Using the proposed framework, improved performance in head pose angle estimation is achieved.

The paper by F. M. Bui and D. Hatzinakos presents biometric methods for secure communications in body sensor networks. The privacy and security arrangements are particularly important in this case since body sensor networks allow pervasive monitoring of potentially sensitive medical data. Two complementary methods are presented based on resource-efficient key management and signallevel data scrambling. Using computer simulations, it is shown that they are very appropriate for delivering secure communications in body sensor networks.

The paper by A. Zimmer and L. Ling presents an offline signature verification system based on online data. In this paper, the online reference data, acquired through a digitizing tablet, serve as the basis for the segmentation process of the corresponding scanned offline data. Local windows are built over the image through a self-adjustable learning process and are used to focus the feature extraction step. In this way, local features are extracted and used by the classifier in combination with global primitives.

The next two papers present biometric systems based on signals captured from the human body. The paper by Y. Wang et al. presents a biometric system based on the analysis of human electrocardiograms. The paper develops a methodology based on existing evidence that human heartbeat could be used as a "difficult-to-falsify" biometric trait. The paper introduces a fiducial detection-based framework that incorporates analytic and appearance attributes. The appearancebased approach needs detection of one fiducial point only. The second paper by A. Riera et al. describes an unobtrusive biometric system based on electroencephalogram analysis. The paper is based on the fact that features extracted from electroencephalogram (EEG) recordings have proved to be unique enough across subjects for biometric applications. A rapid and unobtrusive authentication method is presented that uses only two frontal electrodes and one reference placed at the ear lobe. The resulting matching performance indicates an improvement over that of similar systems presented in an earlier work.

The next three papers deal with problems relevant to identification based on the human eye. The first paper, by H.-A. Park et al., is a study on iris localization and recognition using mobile phones. This paper presents a new iris recognition method for mobile phones based on corneal 
specular reflections (SRs). Novel methods are introduced for (i) the accurate detection of SRs on the cornea of users with glasses, (ii) the robust detection of SRs using eye, camera, and illumination models, and (iii) the verification of the detected iris region. Experimental results based on facial images captured from persons with a mobile phone camera indicate very high rates of correct iris detection. The second paper, by $\mathrm{H}$. Farzin et al., presents a novel retinal identification system. This system comprises of three principal modules including blood vessel segmentation, feature generation, and feature matching. The position and the orientation of the extracted vessels in each scale are used to define a feature vector for each subject in the database. For matching these features across images, a modified correlation measure is introduced in order to obtain a similarity index for each scale of the feature vector. The third paper, authored by J. Wu et al., presents a method for the simultaneous eye tracking and blink detection with interactive particle filters. To this end, two interactive particle filters are used, one for closed eyes and the other for open eyes. Each particle filter is used to track the eye locations and the scales of the eye subjects. Experimental evaluation confirms the utility of the proposed algorithm.

The last paper, by F. Benhammadi et al., presents a fingerprint recognizer using an interminutiae binary constraint graph. The proposed matcher exploits the absolute minutiae features represented as a binary constraint graph. A dynamic tolerance area in the neighborhood of the minutiae, combined with the search-rearrangement method on the binary constraint graph, is used to perform the global matching. Experimental evaluations suggest that the proposed method is promising.

\section{ACKNOWLEDGMENTS}

The guest editors would like to thank the authors who contributed to the present collection of high-quality papers. They are also very grateful to the reviewers for scrutinizing the submitted manuscripts and for suggesting modifications that enhanced the quality of the accepted manuscripts. Last, but certainly not least, they would like to thank the Editorin-Chief and the Editorial Office of EURASIP Journal on Advances in Signal Processing for their continuous efforts and support.

Nikolaos V. Boulgouris Juwei Lu Konstantinos N. Plataniotis Arun Ross 